\title{
THE AGE DISTRIBUTION FUNCTION OF STAR CLUSTERS IN THE LMC
}

\author{
M. ALONGI, C. CHIOSI \\ Department of Astronomy \\ Vicolo dell'Osservatorio 5 \\ I-35122 Padova \\ Italy
}

\begin{abstract}
We present the age distribution function for Large Magellanic Cloud (LMC) clusters obtained from a relationship between their integrated $U B V$ colours and age. We discuss further the results by Chiosi et al. (1988) on the nature of the bimodal distribution of the $(B-V)$ colours for LMC clusters. Their conclusion that bimodality in the colour stems from the combined effect of the age distribution function and the rate at which clusters change colour with age, is revisited. The result of our analysis shows that the age distribution is the sole agent and that a real age gap is present. Finally, we draw some conclusions about the efficiency of cluster formation and disruption in the LMC.
\end{abstract}

The ages of quite a number of LMC clusters have been determined from their integrated $U B V$ colours (Alongi \& Chiosi 1990). The theoretical background relies on the Chiosi et al. (1988) study of the integrated colours of a star cluster as a function of the age and nature of the bimodal distribution of the integrated (B-V) colours for the LMC clusters (van den Bergh, 1981). Chiosi et $a l$. pointed out that, contrary to previous suggestions, the $(B-V)$ bimodality is caused mainly by the age distribution function (ADF) of the star clusters and, partly, by the rate (or colour speed CS) at which a cluster changes its colour as a function of the age. This latter effect is potentially large when the cluster suddenly reddens due to the appearance of either the asymptotic giant branch or the red giant branch (see Chiosi et al. 1988 for details). Both transitions are known to occur on a very short time scale. With the aim of disentangling the two effects, we looked in great detail at the ADF. If the CS is supposed to be the sole cause of bimodality there would be a sudden, large colour-change over a small age interval. It follows from this that the ADF derived from the integrated colours is expected to show no gaps of any type (Alongi \& Chiosi 1990). On the contrary, the age-colour distribution of 137 clusters (193 after correction for completeness) shows two well-separated peaks, each corresponding to $15 \%$ of the population. In terms of age, the gap corresponds to an interval between $T=1.6 \times 10^{8} \mathrm{yr}$ and $t=10^{9} \mathrm{yr}$. This is also visible in the equivalent description of the ADF shown in Fig. 1., which plots the number of clusters per age bin of $\Delta \log (t)=2$. For comparison, the ADF for galactic open clusters (Wielen 1971) is also shown. This latter is normalized to the blue side of the distribution determined for LMC clusters. Comparison of the two distributions lets us derive some conclusions about the efficiency of the processes of cluster formation and disruption in the LMC. Wielen's (1971) distribution is interpreted supposing a constant rate of cluster formation (CFR), so that its decreasing run with age substantially reflects the effects of cluster disruption. Therefore, differences in the ADF for the LMC clusters with respect to the galactic one are likely to reflect the combined effect of a different CFR and disruption law. Even with the large uncertainty associated, the distribution for LMC clusters shows two well-defined peaks, corresponding to $\sim 2.1 \mathrm{Gyr}$ and $\sim 0.1 \mathrm{gyr}$ ago. As expected, this age range coincides roughly with that indicated by the colour-age distribution. 
Furthermore, $2.1 \mathrm{gyr}$ ago is not too far from the value at which, according to Mateo et al. (1990), a sudden enhancement in the rate of star formation (SFR) of field stars took place in the LMC. Mateo et al., studying the C-M diagrams and luminosity functions of several selected samples of field stars, estimate the age of this event about $3 \div 4 \mathrm{Gyr}$ ago. Therefore, we suggest that at least two bursts of cluster formation are present in the ADF, which appear as peaks superposed to a mean declining curve similar, in shape, to the Wielen (1971) one. In turn, the corresponding dips with respect to the mean line could be attributed both to a less efficient CFR and to enhancement in the cluster disruption law. Looking at the detailed morphology of the ADF and at the results for the field stars we may try to tighten them up coherently. The mean slope of the ADF reflects the incidence of disruption, being steeper when the time-averaged disruption rate is greater, while the chronology of the glitches may not be accidental. The chronology of events is: the deep local minimum at about 5 gyr ago is followed by a local maximum around 2 Gyr ago. Between them a burst in the SFR of the field stars occurred, in the range 3+4 Gyr ago according to Mateo et al. (1990) or 3 $\div 5$ Gyr ago according to Frogel \& Blanco (1983). A much less pronounced minimum is visible at $\sim 0.9 \mathrm{Gyr}$ ago, but its statistical evidence seems very uncertain. Much more recently, another local maximum is present, about $10^{8} \mathrm{yr}$ ago, in agreement with the results by Frogel \& Blanco (1983). If the minimum in the ADF at $~ 5 G y r$ ago is real, the first two events (namely this minimum and the burst in the SFR of field stars) could be tightly related, in the sense that some event (likely external to the LMC) could have induced a sudden disruption of the star clusters, leading to an increase in the number of field stars.

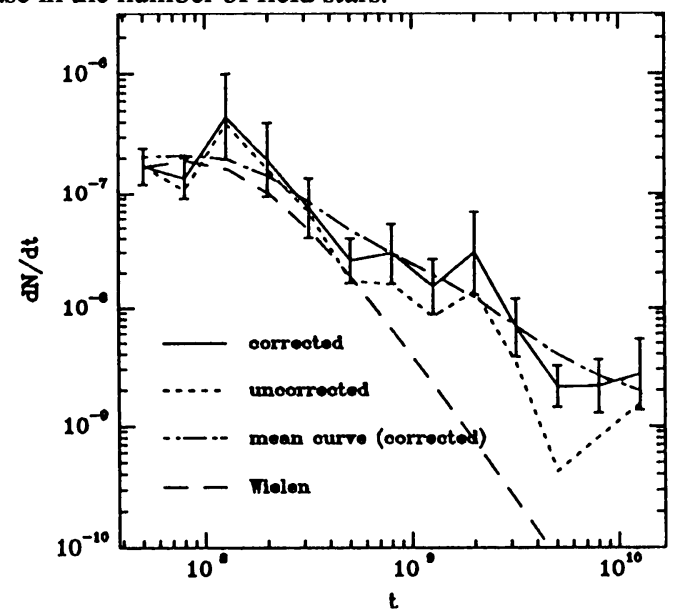

Figure 1. Age distribution for the sample of LMC clusters before (dotted line) and after (solid line) the correction for completeness. The error bars correspond to the values of $\log (\sqrt{ } \Delta N)$, where $\Delta N$ are the clusters falling in each age bin. The dot-dashed line refers to a mean curve corresponding to the corrected sample. The rate for galactic open clusters determined by Wielen (1971) is also shown for comparison. Ages are in years.

\section{References}

Alongi, M., Chiosi, C. (1990), Ap. J. submitted.

Chiosi, C., Bertelli, G., Bressan, A. (1988), Astr. Ap. 196, 84.

Frogel, J., Blanco, V.M. (1983), Ap. J. Lett. 274, L57.

Mateo, M., Bertelli, G., Chiosi, C. (1990), Astrophysical Ages and Dating Methods, E. Vangioni-

Flam, et al. (eds.) (Editions Frontieres: Paris) p.225.

van den Bergh, S. (1981), Astr. Ap. Suppl. 46, 79

Wielen, R. (1971), Astr. Ap. 13, 309. 